\title{
Causal Loop Diagram Model Analysis Approach to the Factors That Influence the SIR Epidemic Model
}

\author{
Renny $^{1, *}$ \\ ${ }^{1}$ Department of Mathematics, Universitas Jenderal Soedirman, Purwokerto, Indonesia \\ *Corresponding author.Email: renny@ unsoed.ac.id
}

\begin{abstract}
Mathematical models can be used to predict the spread of infection of a disease in the future. We developed the SIR model by adding the application of a healthy lifestyle's factor (that related to people's behavior) which is expected can reduce the number of the infected individuals. The analytical approach will be carried out by looking at all the factors that can affect the spread of the disease. We present causal loop diagram (CLD) to explain the relationship among the factors that affect the number of infected individuals of the model. The causal loop diagram helps us in understanding about all the factors that affect the SIR epidemic model and see how these factors are related. We get the result based on the parameters that we used in the mathematical model, that as the level of people awareness, c, increased, the susceptible population gradually increase and the infected population decreases.
\end{abstract}

Keywords: SIR model, system dynamics, Causal Loop Diagram.

\section{INTRODUCTION}

Epidemic model is describing the transmission of infectious disease through individuals. One of the epidemic models is the SIR model which divides the population into three compartment, namely Susceptible, Infected and Recovered. This epidemic model was introduced in 1927 by Kermack and McKendrick that have been derived the initial SIR model [2]. Many variations of the original SIR model have been studied in recent years in order to get more accurate models. That modified SIR models also have been solved by using several different methods. For instance the infected population in [5] is divided into two sub-compartment, and the susceptible population will be the exposed population with certain exposed time, because some of the diseases have their own exposed time to be infected population. In the other cases, [4] have studied the deterministic SEIRS epidemic model for modeling vital dynamics and [3] studied about the stability analysis of SIR model with vaccination.

Causal loop diagram is a simple way to map the interactive elements in feedback systems. The purposed of the causal loop diagram is to demonstrate which element in the dynamic system causes a change in other[8].
In this paper, we discuss the analysis approach to the factors that influence the SIR epidemic models by adding people's behavior parameter. The disease free equilibrium points and endemic equilibrium point of this model will be discussed. We also build the causal loop diagram of the SIR model that gives us some advantages in analysis approach to the model. It will explain the relationship among the factors which affect the number of infected individuals of the model [1]. This causal loop diagram identifies the structures and interactions of feedback loops. It consists of variables that are connected by arrows and denoting the causal influences among the variables.

This paper will be organized as follows: in section 2 , we will discuss about mathematical model of the SIR epidemic model. In section 3, we presented the model in causal loop diagram. In this diagram we explain about the relationship among the factors that influenced the model. In section 4 , we discuss about the analysis of mathematical model. In section 5, we have given the numerical example in support of our result as we discussed in section 2 .

\section{MATHEMATICAL MODEL}

The SIR model is applied by divided population into three compartment, namely, susceptible (S), infected (I), and recovered (R). The model in this paper has the following assumption: 
- The total population is constant.

- The natural birth and death is assumed to be equal.

- All-natural births are into the susceptible compartment.

- style factor, such as level of people awareness to some incident.

- The death rate is equal for all three compartment.

The assumptions lead us to ordinary differential equations system, given by

$$
\begin{aligned}
\mathrm{dS} / \mathrm{dt} & =\mu \mathrm{N}-(\beta-\mathrm{c}) \mathrm{SI} / \mathrm{N}-\mu \mathrm{S} \\
\mathrm{dI} / \mathrm{dt} & =(\beta-\mathrm{c}) \mathrm{SI} / \mathrm{N}-\mu \mathrm{I}-\gamma \mathrm{I} \\
\mathrm{dR} / \mathrm{dt} & =\gamma \mathrm{I}-\mu \mathrm{R}
\end{aligned}
$$

where $\mathrm{S}(\mathrm{t})$ is the number of susceptible people at time $t, I(t)$ is the number of infected people at time $t, R(t)$ is the number of recovered people at time $t, \mu$ is the natural birth and dead rate, $\gamma$ is the recovery rate, $\beta$ is the transmission of disease from an infected people at a time period. The value of $\beta$ will be influenced by the value of $\mathrm{c}$, which is the level of people awareness to some incident. The value of $\mathrm{c}$ can reduce the effective value of the contact rate $\beta$.

The compartment of the model can be described in the following graph as shown in Figure 1.

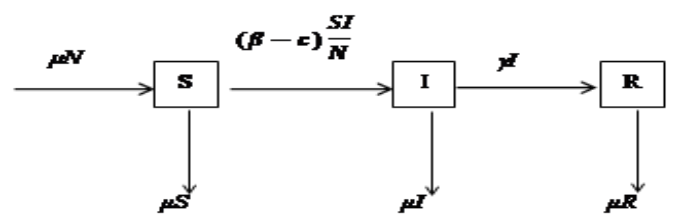

Figure 1 Compartment of SIR model

Since the population is constants, then we consider following equation:

$$
\frac{\mathrm{dN}}{\mathrm{dt}}=\frac{\mathrm{dS}}{\mathrm{dt}}+\frac{\mathrm{dI}}{\mathrm{dt}}+\frac{\mathrm{dR}}{\mathrm{dt}}=0
$$

We used normalization in system as shown in (1), (2), and (3) in order to simplify the variables, so that the system becomes easier to solve. Suppose

and

$$
s=\frac{S}{N}, i=\frac{I}{N}
$$

$$
r=\frac{R}{N}
$$

- In order to decrease the number of infected population, the contact rate between susceptible and infected population will be influenced by the healthy life

where $\mathrm{s}$ is the proportion of the susceptible people to the total population $\mathrm{N}, \mathrm{i}$ is the proportion of the infected people to the total population $\mathrm{N}, \mathrm{r}$ is the proportion of the recovery people to the total population $\mathrm{N}$.

Substituted this proportion into (1), (2), and (3), we get

$$
\begin{aligned}
& \frac{\mathrm{d} s}{\mathrm{~d} t}=\mu-(\beta-c) s i-\mu s \\
& \frac{\mathrm{d} i}{\mathrm{~d} t}=(\beta-c) s i-\mu i-j i \\
& \frac{\mathrm{d} r}{\mathrm{~d} t}=\gamma-\mu r
\end{aligned}
$$

with the initial conditions

$$
s(0) \geq 0, i(0) \geq 0, r(0) \geq 0
$$

We have

or

$$
s(t)+i(t)+r(t)=1
$$

$$
r(t)=1-s(t)-i(t)
$$

\section{CAUSAL LOOP DIAGRAM}

In this section we will discussed about causal loop diagram of the model. Causal loop diagram is an important tool to describe the structure of feedback in a system [7]. In this SIR epidemic model, we could use it as a tools to describe the relation among the variables in the model. Look at the following diagram in Figure 2.

Based on Figure 2, we can describe all factors that affect the SIR epidemic model.

Loop B1 is susceptible population loop; more number of susceptible people will affect more people will be the number of people being infected by the disease, depend on how effective the contact rate in the population, so that they can be infected people. The higher level of people awareness $\mathrm{c}$ will affect to decrease the value of the disease transmission.

Loop B2 is infected population loop; more number of infected people will affect more people lead to death if the contact rate increases and the recovery rate decreases.

Loop B3 is recovered population loop; the number of recovery people will increases depend on the infected people have been recover from the disease. 


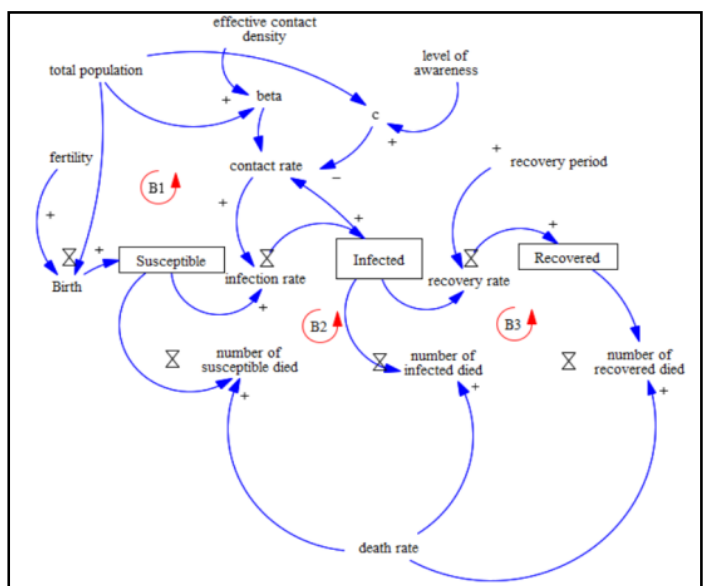

Figure 2 Causal loop diagram of SIR model

From Figure 2, we can determine that: the number of birth is fertility $\mathrm{x}$ total population, infection rate is number of susceptible $\mathrm{x}$ contact rate, which is contact rate is $(\beta-c) x$ number of infected. The value of $c$ is level of people awareness / total population. The value of parameter $\beta$ is effective contact density / total population. Therefore, these value can be used in predicting the infectious people in the population. We will use these parameter simulation value in numerical simulation section.

\section{ANALYSIS OF EQUILIBRIUM POINTS}

As we did in section 1 about mathematical model, we have already normalized system equations (1), (2), and (3) become (5), (6), and (7).

Disease free equilibrium point in (5), (6), and (7) defined as E0 is obtained when there is no infected people in a population or $i=0$. Because we have already known that

$$
r(t)=1-s(t)-i(t)
$$

Therefore it is enough to consider

$$
\begin{aligned}
& \frac{\mathrm{d} s}{\mathrm{~d} t}=\mu-(\beta-c) s i-\mu s \\
& \frac{\mathrm{d} i}{\mathrm{~d} t}=(\beta-c) s i-\mu i-j i
\end{aligned}
$$

Substituted $\mathrm{i}=0$ into nullclines equations, then we have s-nullcline equation,

$$
\begin{aligned}
& \quad \mu-(\beta-c) s i-\mu s=0 \\
& \Leftrightarrow \mu-0-\mu s=0 \\
& \Leftrightarrow s=1
\end{aligned}
$$

Then we have the disease free equilibrium point as $E_{0}$ $=(s, i)=(1,0)$.

In order to get the endemic equilibrium point, E1, we can obtain i-nullcline equation as follows

$$
(\beta-c) s i-\mu i-\gamma i=0
$$

$$
\begin{aligned}
& \Leftrightarrow i((\beta-c) s-\mu-\gamma)=0 \\
& \Leftrightarrow i=0 \text { or }(\beta-c) s-\mu-\gamma=0 \\
& \Leftrightarrow i=0 \text { or } s=\frac{\mu+\gamma}{\beta-c}
\end{aligned}
$$

Substituted

$$
s=\frac{\mu+\gamma}{\beta-c}
$$

into (8), then we have

$$
i=\frac{\mu((\beta-c)-(\mu+\gamma))}{(\beta-c)(\mu+\gamma)}
$$

The endemic equilibrium point is as follows

$$
E_{1}=(s, i)=\left(\frac{\mu+\gamma}{\beta-c}, \frac{\mu((\beta-c)-(\mu+\gamma))}{(\beta-c)(\mu+\gamma)}\right)
$$

The rate of spread of an infectious disease is usually measured by using reproduction number, R0. Reproduction number is the number of secondary infection occurring from the primary infection. In order to get the value of R0, because we want to control the population that can spread the disease, then we need the equation of infected population, $i$.

Suppose $A$ is derivative of $\mathrm{di} / \mathrm{dt}$ respect to $\mathrm{i}$, where $A=M-D$, then $\mathrm{R}_{0}=M D^{-1}[6]$. We know that

$$
A=\frac{d}{d i}\left(\frac{d i}{d t}\right)=(\beta-c) s-\mu-\gamma
$$

Substituted the free disease equilibrium point $(s, i)=$ $(1,0)$, into (10), then

or

$$
A=(\beta-c)(1)-\mu-\gamma
$$

$$
A=(\beta-c)-(\mu+\gamma)
$$

From the previous equation, it can be written that $M=\beta-c$ and $D=\mu+\gamma$. So that,

$$
R_{0}=\frac{\beta-c}{\mu+\gamma}
$$

Endemic equilibrium point exist only when $\mathrm{R}_{0}>1$ or when $\beta-c>\mu+\gamma$, specifically the infection rate must be greater than the recovery rate and the death rate of the infected peoples.

Now we will discuss the linear stability of the equilibrium points by Jacobian.

$$
J(s, i)=\left[\begin{array}{cc}
-(\beta-c) i-\mu & -(\beta-c) s \\
(\beta-c) i & (\beta-c) s-\mu-\gamma
\end{array}\right]
$$

Substituted the equilibrium points $\mathrm{E}_{0}$ into $J(s, i)$,

$$
J(1,0)=\left[\begin{array}{cc}
-\mu & -(\beta-c) \\
0 & (\beta-c)-\mu-\gamma
\end{array}\right]
$$

The characteristic equation corresponding to $\mathrm{E}_{0}$ is

$$
\left|\begin{array}{cc}
-\mu-\lambda & -(\beta-c) \\
0 & (\beta-c)-\mu-\gamma-\lambda
\end{array}\right|=0
$$

Therefore, we get the eigenvalues i.e

$$
\lambda_{1}=-\mu \text { and } \lambda_{2}=(\beta-c)-(\mu+\gamma)
$$

The value of $\lambda_{1}<0$. If $(\beta-c)-(\mu+\gamma)<0$ then $(\beta-c)<(\mu+\gamma)$ or $\frac{(\beta-c)}{\mu+\gamma}<1$ or $R_{0}<1$. 
As both of the eigenvalues are negative, then the disease free equilibrium E0 is locally asymptotically stable.

\section{NUMERICAL SIMULATION}

In this section, we will discuss about the result of the SIR epidemic model by taking a numerical simulation. By taking the parameters value $\mu=0.2$, $\beta=1, c=0.1, \gamma=0.3$, we get the following simulation on Figure 3.

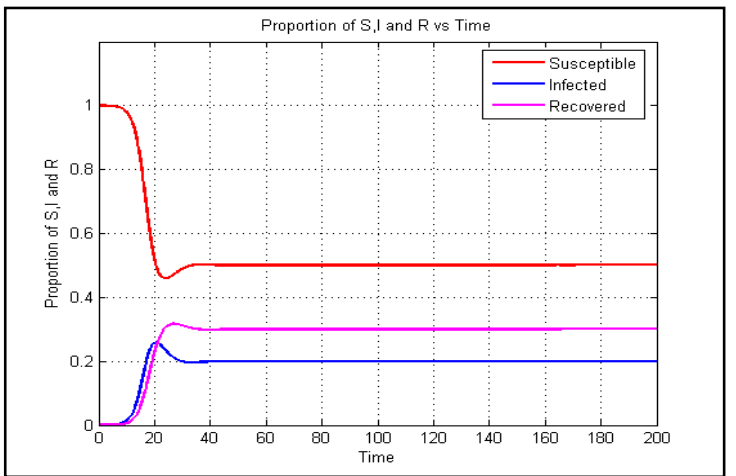

Figure 3 Simulation 1

When we increased the value of $\beta=1.1$, and $c=0.2$, since the value of the other parameter still the same, then we have the endemic equilibrium point is $(0.499$ $, 0.202,0.2995)$ as shown in Figure 4.

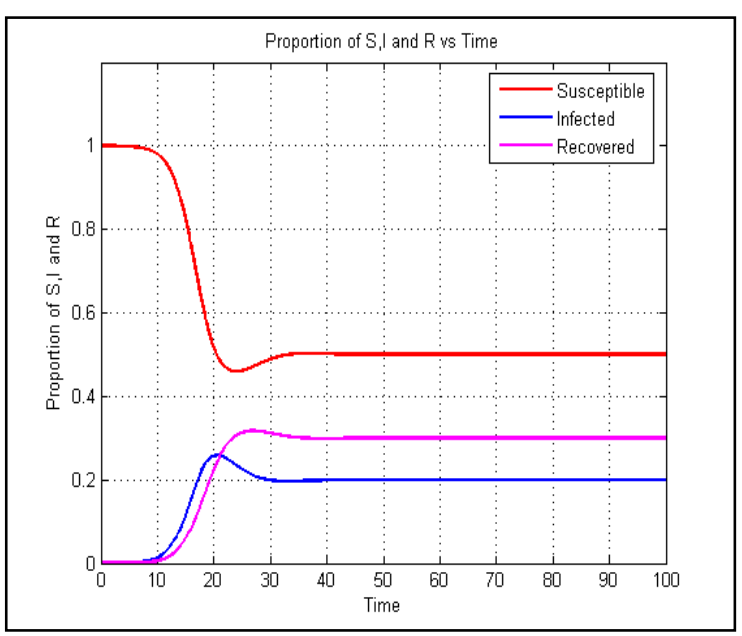

Figure 4 Simulation 2

When we decreased the value of $\beta=0.85$, and the value of the other parameter still the same, then we have the endemic equilibrium point is (0.6671, $0.1332,0.1996)$ as shown in Figure 5.

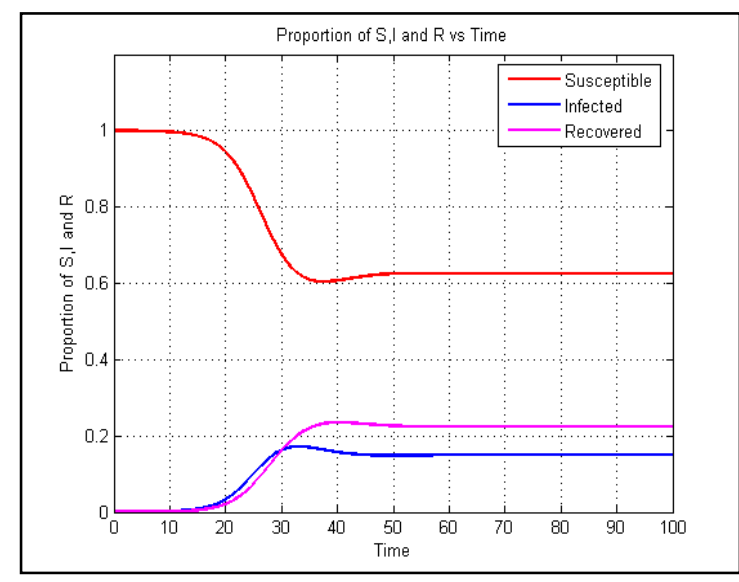

Figure 5 Simulation 3

As the level of people awareness, c, increased, the susceptible population gradually increase and the infected population decreases.

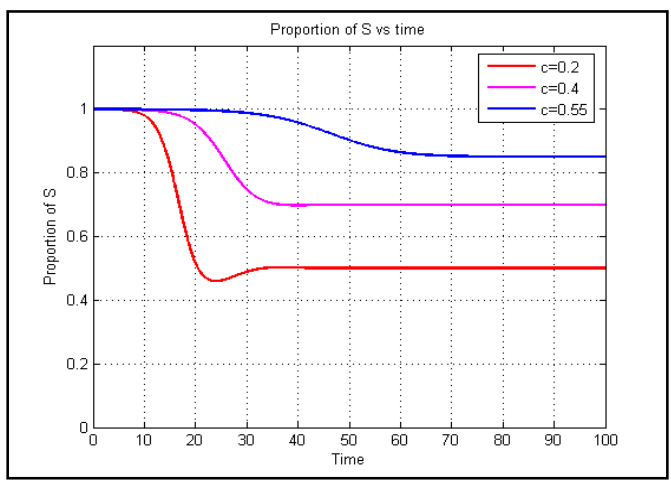

Figure 6 Simulation 4

Let's take a look at the following simulation in Figure 6, when we consider the level of people awareness increased.

Based on the simulation in Figure 7, we can say that the level of people awareness play had a very important role in controlling the spread of a disease.

By doing a random simulation for parameter $\mathrm{c}$, then in Figure 8 described that how a level of people's awareness very affects the number of the susceptible and infected population.

In Table 1, presented random simulation value for people's awareness, that affected the number of the number of the susceptible, infected, and recovered population. We used $\mathrm{t}=0 . .12$. 


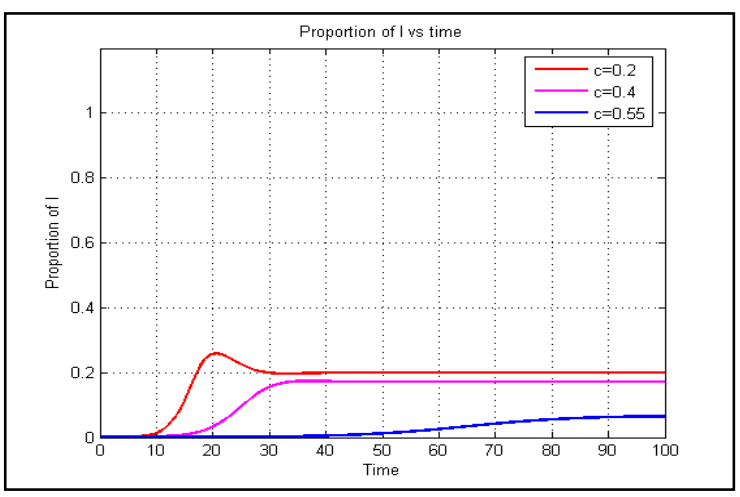

Figure 7 Simulation 5

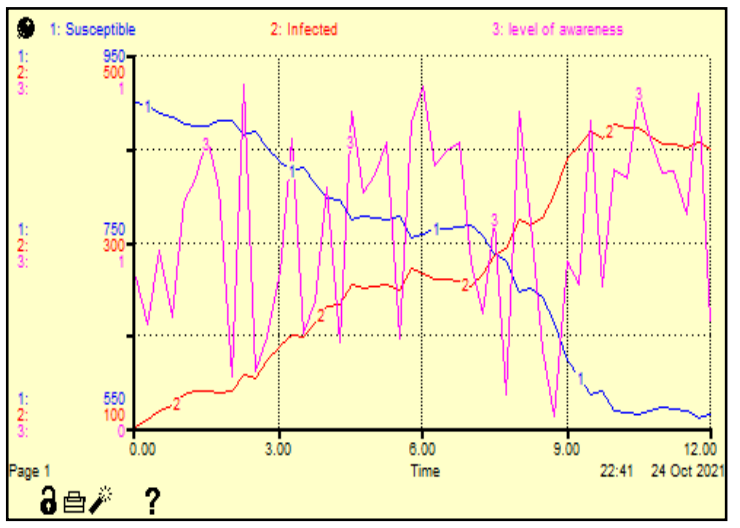

Figure 8 Simulation 6

Table 1. Random simulation value table for the number of the susceptible, infected, and recovered population

\begin{tabular}{|c|c|c|c|c|}
\hline \multirow{2}{*}{ Time } & \multirow{2}{*}{$\begin{array}{c}\text { Proportion } \\
\text { of people's } \\
\text { awareness }\end{array}$} & \multicolumn{3}{|c|}{ Number of } \\
\hline 0.0 & 0.61 & 900 & 100 & 1 \\
\hline 0.3 & 0.88 & 904.7 & 98.1 & 2.9 \\
\hline 0.5 & 0.01 & 909.4 & 95.9 & 5.1 \\
\hline 0.8 & 0.47 & 894.5 & 113.8 & 6.9 \\
\hline 1.0 & 0.78 & 893.6 & 116.8 & 9.5 \\
\hline 1.3 & 0.48 & 898.3 & 114.6 & 11.8 \\
\hline 1.5 & 0.78 & 903.0 & 111.8 & 14.6 \\
\hline 1.8 & 0.78 & 907.7 & 109.6 & 16.8 \\
\hline 2.0 & 0.70 & 912.4 & 107.6 & 18.8 \\
\hline 2.3 & 0.67 & 912.4 & 109.7 & 21.4 \\
\hline 2.5 & 0.77 & 917.5 & 107.3 & 23.8 \\
\hline 2.8 & 0.90 & 921.8 & 104.9 & 26.2 \\
\hline 3.0 & 0.72 & 926.6 & 102.6 & 28.5 \\
\hline 3.3 & 0.67 & 931.3 & 100.7 & 30.4 \\
\hline
\end{tabular}

\begin{tabular}{|c|c|c|c|c|}
\hline \multirow{2}{*}{ Time } & \multirow{2}{*}{$\begin{array}{l}\text { Proportion } \\
\text { of people's } \\
\text { awareness }\end{array}$} & \multicolumn{3}{|c|}{ Number of } \\
\hline 3.5 & 0.96 & 935.6 & 99.3 & 32.2 \\
\hline 3.8 & 0.85 & 940.3 & 97.3 & 34.2 \\
\hline 4.0 & 0.11 & 945.1 & 94.9 & 36.6 \\
\hline 4.3 & 0.52 & 933.1 & 109.5 & 38.7 \\
\hline 4.5 & 0.40 & 929.6 & 115.0 & 41.3 \\
\hline 4.8 & 0.81 & 934.3 & 112.2 & 44.2 \\
\hline 5.0 & 0.40 & 939.0 & 110.0 & 46.3 \\
\hline 6.0 & 0.95 & 954.5 & 104.1 & 55.6 \\
\hline 7.0 & 0.85 & 953.6 & 115.5 & 64.1 \\
\hline 8.0 & 0.08 & 951.4 & 126.7 & 73.9 \\
\hline 9.0 & 0.07 & 954.8 & 131.0 & 85.1 \\
\hline 10.0 & 0.39 & 905.0 & 187.5 & 97.3 \\
\hline 11.0 & 0.08 & 883.0 & 212.2 & 113.5 \\
\hline 12.0 & 0.60 & 828.5 & 268.2 & 130.9 \\
\hline
\end{tabular}

\section{CONCLUSIONS}

In this paper, we discussed about how the effect of level people awareness has given an important affect to the number of susceptible and infected in population. As the level of people awareness, c, increased, the susceptible population gradually increase and the infected population decreases.

The causal loop diagram helps us in understanding about all the factors that affect the SIR epidemic model and see how these factors are related.

For future research, we can convert the causal loop diagram into stock flow diagram to present all the details of the model. We use the stock flow diagram for simulation and sensitivity analysis of the model.

\section{ACKNOWLEDGMENT}

The author expresses the gratitute and sincere appreaciation to all parties that involved in this research. This research was funded by Lembaga Penelitian dan Pengabdian Masyarakat Universitas Jenderal Soedirman (Riset Institusional Unsoed Scheme No. T/458/UN23.18/PT.01.03/2021).

The author also want to thank to the anonymous reviewers for their valuable comments to revise this paper. 


\section{REFERENCES}

[1] A. Kumar, B. Priya, and S.K. Srivastava, "Response to the COVID-19: Understanding implications of government lockdown policies," Journal of Policy Modeling, vol. 43, pp. 76 - 94, January-February 2021.

[2] N. Bacaër, "A short historical of mathematical population dynamics," pp. 89 - 96, SpringerVerlag London, 2011.

[3] S. Chauhan, O.P. Misra and J. Dhar, "Stability analysis of SIR model with vaccination," American Journal of Computational and Applied Mathematics," Vol. 1, pp. 17 - 23, 2014.

[4] M.B. Trawicki, "Deterministic SEIRS epidemic model for modeling vital dynamics, vaccination, and temporary immunity," Mathematics, Vol. 5(1), 2017.

[5] N.R. Sasmita, M. Ikhwan, S. Suyanto, and V. Chongsuvivatwong, "Optimal control on a mathematical model to pattern the progression of coronavirus disease 2019 (COVID-19) in Indonesia," Global Health Research and Policy, Vol 5(38), 2020.

[6] K.Q. Fredlina, T.B. Oka and I.M.E. Dwipayana, "SIR model for the spread of Tuberculosis disease," Jurnal Matematika, Vol. 1(1), pp. 52 58, 1 August 2012

[7] D. Nuryadin, S.Saleh, A.S.Hardi, E.H. Pangaribowo, "Developing a dynamic model for sustainable development in Yogyakarta city," The Indonesian Journal of Planning and Development, Vol. 4(2) pp. 57-68, October 2019.

[8] A.Z. Senaras, "Causal loop diagrams and feedbacks: A case study in flexible manufacturing system," Yönetim, Ekonomi ve Pazarlama Arastirmalari Dergisi, Vol 1(5), pp.112, 2017. 